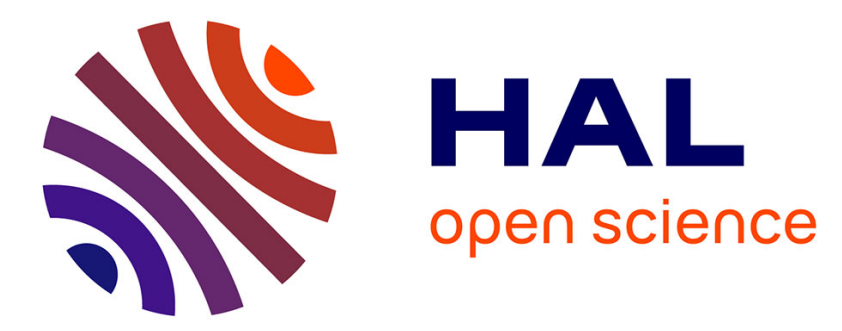

\title{
Generation and conversion of optical vortices in long-period twisted elliptical fibers
}

Constantine N. Alexeyev, Tatiana A. Fadeyeva, Boris P. Lapin, Maxim A. Yavorsky

\section{- To cite this version:}

Constantine N. Alexeyev, Tatiana A. Fadeyeva, Boris P. Lapin, Maxim A. Yavorsky. Generation and conversion of optical vortices in long-period twisted elliptical fibers. Applied optics, 2012, 51 (10), pp.C193-C197. 10.1364/AO.51.00C193 . hal-00731812

\section{HAL Id: hal-00731812 \\ https://hal.science/hal-00731812}

Submitted on 13 Mar 2018

HAL is a multi-disciplinary open access archive for the deposit and dissemination of scientific research documents, whether they are published or not. The documents may come from teaching and research institutions in France or abroad, or from public or private research centers.
L'archive ouverte pluridisciplinaire HAL, est destinée au dépôt et à la diffusion de documents scientifiques de niveau recherche, publiés ou non, émanant des établissements d'enseignement et de recherche français ou étrangers, des laboratoires publics ou privés.

\section{(ㅇ)(1) 80}

Distributed under a Creative Commons Attribution - NonCommercial - ShareAlikel 4.0 


\title{
Generation and conversion of optical vortices in long-period twisted elliptical fibers
}

\author{
Constantine N. Alexeyev, ${ }^{1, \star}$ Tatiana A. Fadeyeva, ${ }^{1}$ \\ Boris P. Lapin, ${ }^{1}$ and Maxim A. Yavorsky ${ }^{1,2}$ \\ ${ }^{1}$ Taurida National V.I. Vernadsky University, Vernadsky Prospekt, 4, Simferopol, 95007, Crimea, Ukraine \\ 2Univ. Bordeaux, LOMA, UMR 5798, F-33400 Talence, France; CNRS, LOMA, UMR 5798, F-33400 Talence, France \\ ${ }^{*}$ Corresponding author: c.alexeyev@yandex.ua
}

\begin{abstract}
We theoretically demonstrate that long period twisted elliptical fibers have the ability to change in a certain wavelength range the topological charge of the incoming field by two units. We also show that such fibers can generate charge 2 optical vortices from the incoming Gaussian beams.
\end{abstract}

OCIS codes: $\quad 060.2280,060.2400,060.2310,260.6042$.

\section{Introduction}

Generation of optical fields with singularities has become a topical problem of singular optics since first discussions of optical vortices (OVs) [1,2]. At present, several methods of OV generation are used for such a purpose: generation by lens converters [3], spiral phase plates [4], and phase holograms [5]. In past years other new methods have been suggested $[\underline{6}, 7,8]$. The method of OV generation by so-called $q$-plates has shown great promise $[9,10]$.

Among the variety of the existing methods, one can single out the special group concerned with OV generation with optical fibers. Though some of them exploit the semblance of stress-applied fibers with lens converters [11], the majority are based on the effect of mode coupling in chiral fiber gratings. Historically, first such an effect of vortex generation (without its recognition) from the Gaussian mode was presented in [12]. Similar phenomena have been observed in other types of helical fiber gratings [13-15]. A theoretical explanation of the observed results, though, has been presented quite recently $[2,16,17]$. In those papers it has been pointed out that a helical pertur- bation of refractive index brings forth the coupling between fiber modes with orbital numbers differing by unity. The effect of such mode coupling is insensitive to a particular technique of creation of a helical perturbation and leads to changing the topological charge of the incident field by unity [18]. At present such helical-core fibers are no longer some bizarre objects but are within the reach of state of the art technology $[19,20]$.

However, all such waveguides with a helical perturbation of refractive index have a common limitation: they can change the topological charge of the incoming field only by unity. Meanwhile, it is desirable to have the possibility of changing this charge in somewhat wider limits. In this paper we propose the method of all-fiber changing the topological charge of the incoming field by two units. An inspiring hint on the nature of the class of fibers, which could be the candidates for such systems, can be found in the papers of Kopp et al. on twisted fibers. In one of their early papers on that topic the authors refer to effectively elliptic twisted fiber as to a double-helix fiber [21]. Indeed, in a way, such fibers feature $\pi$-shifted helices of larger refraction index $n$. Though, generally speaking, actual distribution of $n$ is more complicated, this notion proves to be sufficient to focus attention on such class of fibers. 
The aim of this paper is to demonstrate that longperiod twisted elliptic fibers can change the topological charge of the incoming field by two units. In particular, we show that such fibers can generate charge-2 OV from the incoming Gaussian beams.

\section{Basic Equations and Coupled Modes}

Elliptical twisted fibers are manufactured by simultaneously drawing and twisting the fiber from a preform with an elliptically deformed core. During such technological process no elastic strains appear in the fiber and the effect of twisting is reduced to a mere geometrical modification of refractive index distribution. As is shown in [22], for weakly guiding fibers this leads to the following distribution of the refractive index:

$$
\begin{aligned}
n^{2}(r, \varphi, z)= & n_{\mathrm{co}}^{2}(1-2 \Delta f(r)) \\
& -2 n_{\mathrm{co}}^{2} \Delta \delta r f_{r}^{\prime} \cos (2(\varphi-q z)),
\end{aligned}
$$

where $\Delta$ is the height of the profile $f, \delta \ll 1$ is dimensionless parameter of ellipticity, $n_{\text {co }}$ is the core's refractive index, and $q=2 \pi / H$, with $H$ being the pitch of the fiber [see Fig. 1]. Here the axial-polar coordinates $(r, \varphi, z)$ are implied and are introduced in the standard way.

In the scalar approximation, which proves to be sufficient for our purposes, the transverse electric field $\vec{E}_{t}$ satisfies the following equation [23]:

$$
\Delta \vec{E}_{t}+k^{2} n^{2} \vec{E}_{t}=0
$$

where $k$ is the wave number in vacuum and $\Delta$ is the Laplace operator. The change of variables $\tilde{r}=r$, $\tilde{z}=z, \tilde{\varphi}=\varphi-q z$ enables one to obtain the translational invariant in $\tilde{z}$ equation:

$$
\begin{aligned}
\left\{\frac{\partial^{2}}{\partial \tilde{r}^{2}}+\right. & \frac{1}{\tilde{r}} \frac{\partial}{\partial \tilde{r}}+\frac{1}{\tilde{r}^{2}} \frac{\partial^{2}}{\partial \tilde{\varphi}^{2}}+\left(\frac{\partial}{\partial \tilde{z}}-q \frac{\partial}{\partial \tilde{\varphi}}\right)^{2}+k^{2} \tilde{n}^{2}(\tilde{r}) \\
& \left.-2 k^{2} n_{\mathrm{co}}^{2} \tilde{r} \Delta \delta f_{\tilde{r}}^{\prime} \cos 2 \tilde{\varphi}\right\} \vec{E}_{t}=0,
\end{aligned}
$$

which after the substitution $\vec{E}_{t}=\vec{e}_{t}(\tilde{r}, \tilde{\varphi}) \exp$ $(i \beta \tilde{z}), \beta$ being the propagation constant, is reduced to

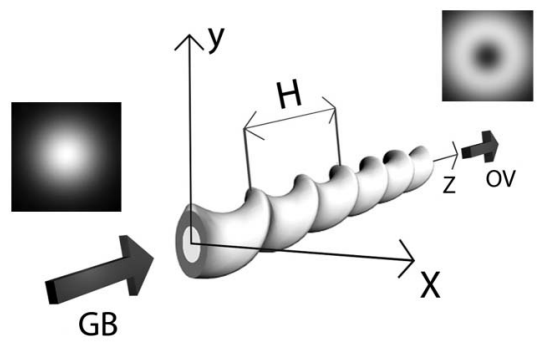

Fig. 1. Geometry of the problem: Schematically shown genera tion of the optical vortex OV from the incident Gaussian beam GB. Insets show intensity distribution of the corresponding fields.

$$
\begin{gathered}
\left\{\frac{\partial^{2}}{\partial \tilde{r}^{2}}+\frac{1}{\tilde{r}} \frac{\partial}{\partial \tilde{r}}+\frac{1}{\tilde{r}^{2}} \frac{\partial^{2}}{\partial \tilde{\varphi}^{2}}+\left(i \beta-q \frac{\partial}{\partial \tilde{\varphi}}\right)^{2}+k^{2} \tilde{n}^{2}\right. \\
\left.-2 k^{2} n_{\mathrm{co}}^{2} \tilde{r} \Delta \delta f_{\tilde{r}}^{\prime} \cos 2 \tilde{\varphi}\right\} \mathbf{e}_{t}(\tilde{r}, \tilde{\varphi})=0
\end{gathered}
$$

In the basis of linear polarizations $|e\rangle=\left(\begin{array}{l}e_{x} \\ e_{y}\end{array}\right)$ this equation can be recast as

$$
\left(\hat{H}_{0}+\hat{V}\right)|e\rangle=\beta^{2}|e\rangle,
$$

where

$\hat{H}_{0}=\left(\frac{\partial^{2}}{\partial \tilde{r}^{2}}+\frac{1}{\tilde{r}} \frac{\partial}{\partial \tilde{r}}+\frac{1}{\tilde{r}^{2}} \frac{\partial^{2}}{\partial \tilde{\varphi}^{2}}+k^{2} \tilde{n}^{2}-2 i \beta q \frac{\partial}{\partial \tilde{\varphi}}+q^{2} \frac{\partial^{2}}{\partial \tilde{\varphi}^{2}}\right)$,

$\hat{V}=-2 k^{2} n_{\mathrm{co}}^{2} \tilde{r} \Delta \delta f_{\tilde{r}}^{\prime} \cos 2 \tilde{\varphi}$. Zero approximation eigenvalue equation $\hat{H}_{0}|e\rangle=\bar{\beta}^{2}|e\rangle$ readily yields eigenvectors given by circularly polarized OVs:

$$
|\sigma, l\rangle=\left(\begin{array}{c}
1 \\
i \sigma
\end{array}\right) \exp (i l \tilde{\varphi}) F_{l}(r)
$$

where $\sigma= \pm 1$ determines the circular polarization, $l=0, \pm 1, \pm 2 \ldots$ is the topological charge of the vortex solution, and radial functions satisfy [23]

$$
\left(\frac{\partial^{2}}{\partial r^{2}}+\frac{1}{r} \frac{\partial}{\partial r}+k^{2} \tilde{n}^{2}-\frac{l^{2}}{r^{2}}-\tilde{\beta}_{l}^{2}\right) F_{l}(r)=0 .
$$

For the spectrum of propagation constants, one obtains

$$
\beta_{l}^{(1,2)}= \pm \tilde{\beta}_{l}+l q
$$

As is seen from Eq. (8), in general, at $q \neq 0$ there is no degeneracy in the spectra (at $q=0$ one should take account of the vector term in the waveguide equation [22]). However, at certain points the curves plotted as functions of $q$ may intersect. In such points of the so-called accidental degeneracy, one has to use the perturbation theory with degeneracy to allow for the influence of the perturbation term $\hat{V}$ in Eq. (5). We will demonstrate the application of this technique at the example of $l=0,2$ families of spectral curves.

Since the perturbation term $\hat{V}$ cannot provide any coupling of fields with opposite polarizations, it is sufficient to study only spectral curves of zeroapproximation modes of the same polarization. The spectra of $\sigma=1$ modes at $l=0,2$ are

$\beta_{1,2}= \pm \tilde{\beta}_{0}, \quad \beta_{3,4}= \pm \tilde{\beta}_{2}+2 q, \quad \beta_{5,6}= \pm \tilde{\beta}_{2}-2 q$

The plots of these curves are given in Fig. 2 . At the points (a) and (b) (at $\left.q=q_{0} \equiv\left(\tilde{\beta}_{0}-\tilde{\beta}_{2}\right) / 2\right)$ the curves of $l=0$ and $l=2$ modes intersect. In such 


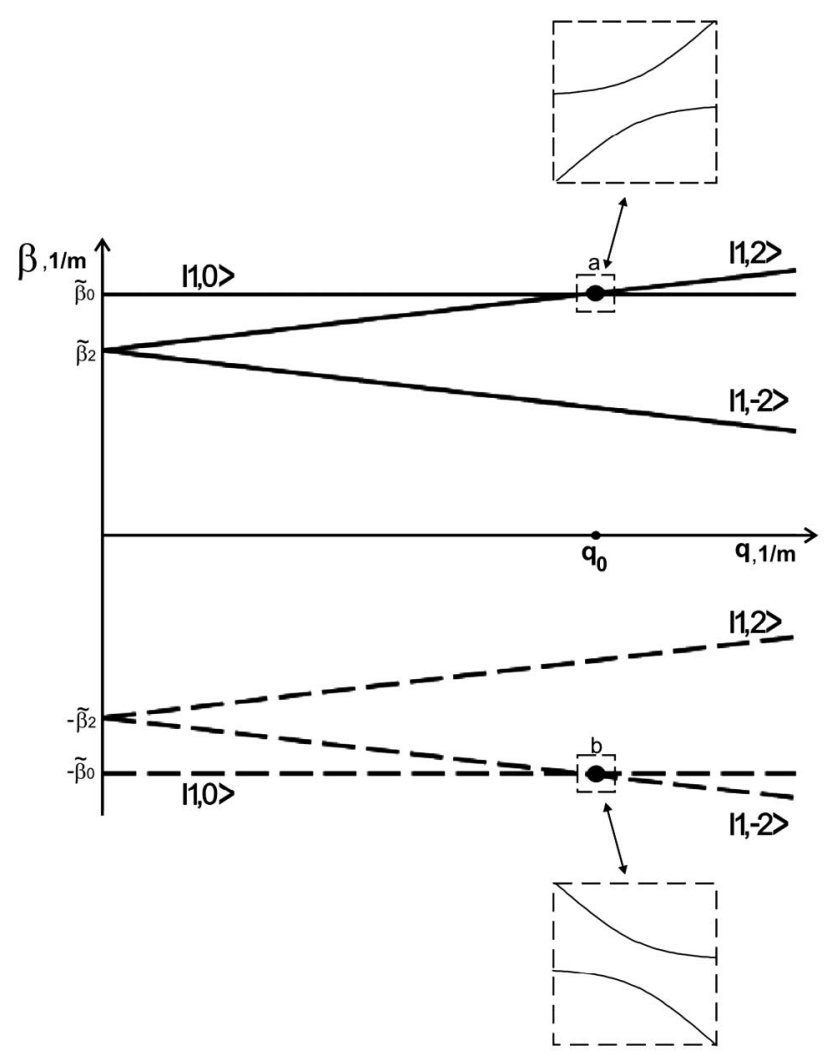

Fig. 2. Zero approximation spectra of twisted elliptical fiber modes versus lattice vector $q$. The type of the mode is indicated at the corresponding curve. Insets show repulsion of spectral branches due to the effect of mode coupling; the fiber's parameters are: $n_{\text {co }} 1.5, \Delta \quad 0.01, \delta \quad 0.05, r_{0} \quad 8 \lambda_{0}, \quad \lambda_{0} 632.8 \mathrm{~nm}$, $q \approx q_{0} \quad 11216.845 \mathrm{~m}^{-1}$.

points there takes place intensive hybridization of forward-propagating (a) and backward-propagating (b) zero-approximation modes. For example, at point (a), forward-propagating modes $|1,0\rangle$ and $|1,2\rangle$ get coupled, whereas other modes do not interact. According to [24], to obtain the exact form of coupled modes, one has to build the matrix of the total Hamiltonian $\hat{H}_{0}+\hat{V}$ in the basis of those zeroapproximation eigenvectors of $\hat{H}_{0}$, whose spectra coincide at $q=q_{0}$. Then the eigenvector problem is reduced to $[\underline{3}, \underline{16}]$

$$
\left(\begin{array}{cc}
\tilde{\beta}_{0}^{2}-\beta^{2} & A \\
A & \tilde{\beta}_{2}^{2}-(\beta-2 q)^{2}
\end{array}\right) \vec{x}_{a}=0
$$

where $A=-k^{2} n_{\text {co }}^{2} \Delta \delta / N_{0} N_{2}$, normalization factor $N_{i}=\sqrt{ } \int_{0}^{\infty} x F_{i}^{2} \mathrm{~d} x$. Here the vector $\vec{x}_{a}=\left(x_{1}, x_{2}\right)$ corresponds to the field $x_{1}|1,0\rangle+x_{2}|1,2\rangle$. Introducing detunings $\varepsilon=q-q_{0}$ and $\delta=\beta-\tilde{\beta}_{0}$, one can further simplify the eigenvalue problem:

$$
\left(\begin{array}{cc}
2 \tilde{\beta}_{0} \delta & A \\
A & 2 \tilde{\beta}_{2}(2 \varepsilon-\delta)
\end{array}\right) \vec{x}_{a}=0
$$

The spectra feature the so-called repulsion of spectral curves [24] (see insets in Fig. 2) and are given by the expressions

$$
\begin{aligned}
& \beta_{1,2}^{(a)}=\tilde{\beta}_{0}+\varepsilon \pm \sqrt{ } \varepsilon^{2}+\Gamma^{2}, \\
& \beta_{1,2}^{(b)}=-\tilde{\beta}_{0}-\varepsilon \pm \sqrt{ } \varepsilon^{2}+\Gamma^{2},
\end{aligned}
$$

where $\Gamma^{2} \approx A^{2} / 4 \tilde{\beta}_{0}^{2}$. After a little algebra, one can obtain the expressions for coupled modes:

$$
\begin{aligned}
\left|\Psi_{1 a}\right\rangle= & \left\{c_{1}|1,0\rangle \exp \left[i\left(\tilde{\beta}_{0}+\varepsilon\right) z\right]+c_{2}|1,2\rangle \exp \left[i\left(\tilde{\beta}_{2}-\varepsilon\right) z\right]\right\} \\
& \times \exp \left(i z \sqrt{ } \varepsilon^{2}+\Gamma^{2}\right) \\
\left|\Psi_{2 a}\right\rangle= & \left\{-c_{2}|1,0\rangle \exp \left[i\left(\tilde{\beta}_{0}+\varepsilon\right) z\right]+c_{1}|1,2\rangle\right. \\
& \left.\times \exp \left[i\left(\tilde{\beta}_{2}-\varepsilon\right) z\right]\right\} \exp \left(-i z \sqrt{ } \varepsilon^{2}+\Gamma^{2}\right)
\end{aligned}
$$

where $c_{1,2}=\frac{1}{\sqrt{ } 2} \sqrt{ } 1 \mp \frac{\varepsilon}{\sqrt{\varepsilon^{2}+\Gamma^{2}}}$. Analogously, one obtains the formulae for coupled backward-propagating modes:

$$
\begin{aligned}
\left|\Psi_{1 b}\right\rangle= & \left\{-c_{2}|1,0\rangle \exp \left[-i\left(\tilde{\beta}_{0}+\varepsilon\right) z\right]+c_{1}|1,-2\rangle\right. \\
& \left.\times \exp \left[-i\left(\tilde{\beta}_{2}-\varepsilon\right) z\right]\right\} \exp \left(i z \sqrt{ } \varepsilon^{2}+\Gamma^{2}\right), \\
\left|\Psi_{2 b}\right\rangle= & \left\{c_{1}|1,0\rangle \exp \left[-i\left(\tilde{\beta}_{0}+\varepsilon\right) z\right]+c_{2}|1,-2\rangle\right. \\
& \left.\times \exp \left[-i\left(\tilde{\beta}_{2}-\varepsilon\right) z\right]\right\} \exp \left(-i z \sqrt{ } \varepsilon^{2}+\Gamma^{2}\right) .
\end{aligned}
$$

It should be emphasized that the fields in Eqs. (13) depend on the azimuthal coordinate $\varphi$ and not on $\tilde{\varphi}$. The remaining OVs: backward-propagating $|1,2\rangle$ and forward-propagating $|1,-2\rangle$, remain uncoupled and their fields do not alter. The results obtained are sufficient to solve the problem of Gaussian mode's passage through such a fiber.

\section{Generation of Double-Charged Optical Vortices}

Let us study now the passage of the Gaussian beam through the twisted elliptical fiber with $q=q_{0}$. If the waist radius of the beam is correlated with the core's radius near the input end [23] the incident Gaussian beam can be approximated by $|1,0\rangle$ mode. Before the fiber the field is given by the incident and reflected fields:

$$
\begin{aligned}
\left|\Phi_{1}(z \leq 0)\right\rangle= & |1,0\rangle e^{i k z}+R_{1}|1,0\rangle e^{i k z}+R_{2}|1,2\rangle e^{i k z} \\
& +R_{3}|1,-2\rangle e^{i k z} .
\end{aligned}
$$

Within the fiber, the field can be represented as

$$
\begin{aligned}
& \left|\Phi_{2}\right\rangle=T_{1}\left|\psi_{1 a}\right\rangle+T_{2}\left|\psi_{2 a}\right\rangle+T_{3}\left|\psi_{1 b}\right\rangle+T_{4}\left|\psi_{2 b}\right\rangle \\
& \quad+T_{5}|1,2\rangle e^{i \tilde{\beta}_{2} z}+T_{6}|1,-2\rangle e^{i \tilde{\beta}_{2} z}
\end{aligned}
$$


whereas the output field looks like

$$
\left|\Phi_{3}(z \geq d)\right\rangle=\left(P_{1}|1,0\rangle+P_{2}|1,2\rangle+P_{3}|1,-2\rangle\right) e^{i k(z d)} .
$$

Here $R_{i}, T_{i}$, and $P_{i}$ are unknown coefficients. As usual, the linear algebraic equations for these coefficients are obtained from matching fields and their derivatives with respect to $z$ at the boundaries.

The dependence of transmission coefficients $\left|P_{i}\right|^{2}$ versus wavelength of the incident Gaussian beam is shown in Fig. 3. As follows from numerical results, at certain wavelength range the incident Gaussian beam gets almost completely transformed into charge-2 OV $|1,2\rangle$. As the fiber's length increases, the area of effective conversion diminishes. Figure 4 shows typical conversion curves at $d=207 \mathrm{~mm}$. These results demonstrate that twisted elliptical fibers can be used as all-fiber generators of charge$2 \mathrm{OV}$.

As is evident, this class of fibers has the ability to change the topological charge of the incoming field by 2 units. For example, such fibers can convert an incident $|1,1\rangle$ OV into OV $|1,3\rangle$ [Fig. 5]. Such selectivity can be explained through dynamical properties of the perturbation operator $V \propto \cos 2 \tilde{\varphi}$ : it can couple only the basic vectors $|\sigma, l\rangle$, whose orbital numbers differ by two units: $\langle\sigma, l|\hat{V}| \sigma, l \pm 2\rangle \neq 0$. Of course such conversion would take place at other $q$ or wavelength $\lambda$ than $q_{0}$ and $\lambda_{0}$, where the Gaussian beam gets converted into the OV. Basically, this conversion of the topological charge is closely connected with the

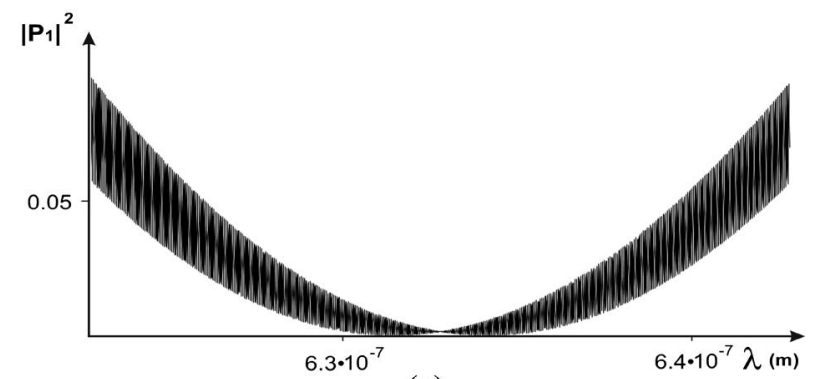

(a)

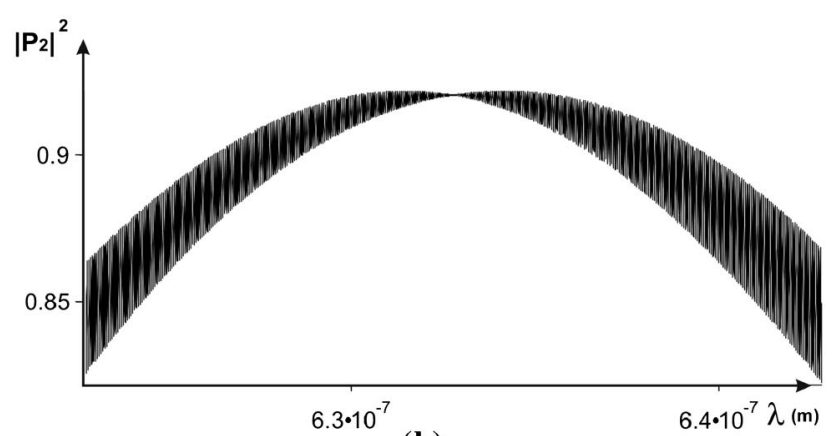

(b)

Fig. 3. Transmission coefficients $\left|P_{i}\right|^{2}$ for the outcoming modes $|1,0\rangle$ (a) and $|1,2\rangle$ (b) versus wavelength of the incoming field $|1,0\rangle$; the fiber's length $d \quad 2.55 \mathrm{~mm}, \Delta \quad 0.01, \delta \quad 0.05, r_{0} \quad 8 \lambda_{0}$, $\lambda_{0} \quad 632.8 \mathrm{~nm}, H \quad 0,56 \mathrm{~mm}$. The coefficient for the outcoming mode $|1,2\rangle$ is negligibly small (not shown).

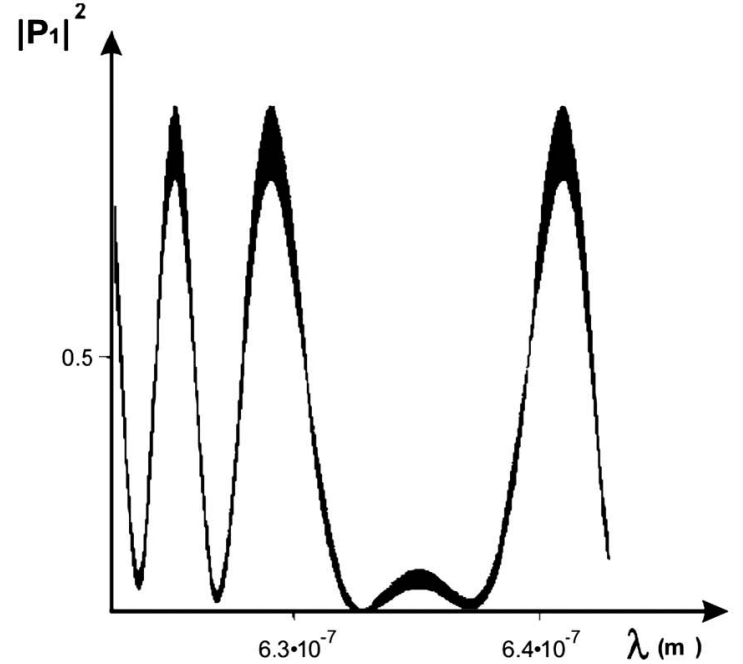

(a)

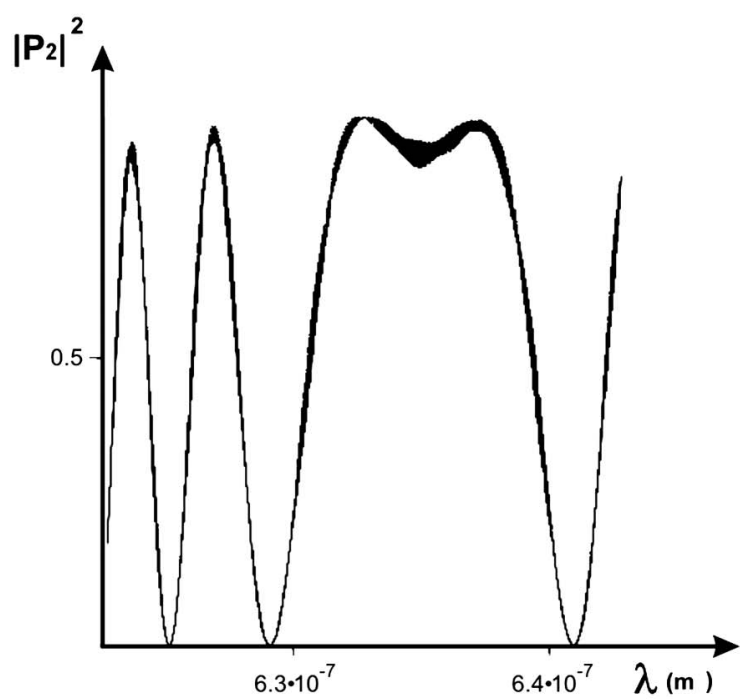

(b)

Fig. 4. Transmission coefficients for the outcoming modes $|1,0\rangle$ (a) and $|1,2\rangle$ (b) versus wavelength of the incoming field $|1,0\rangle$; the fiber's length $d \quad 207 \mathrm{~mm}$. The other parameters are the same as in Fig. 3 .

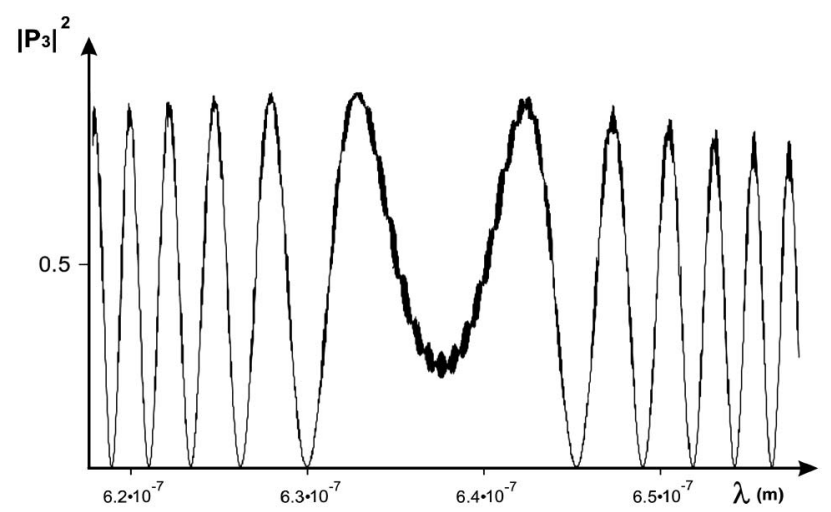

Fig. 5. Transmission coefficient for the outcoming vortex $|1,3\rangle$ versus wavelength of the incoming field $|1,1\rangle$. Fiber parameters: $d \quad 209.3 \mathrm{~mm}, \quad n_{\text {со }} 1.5, \Delta \quad 0.01, \delta \quad 0.05, \quad r_{0} 8 \lambda_{0}, \quad \lambda_{0}$ $632.8 \mathrm{~nm}, H \quad 0.44 \mathrm{~mm}$. 
presence of a double-helix in the structure of lines of equal refractive index.

\section{Conclusion}

In this paper we have theoretically demonstrated that long-period twisted elliptical fibers possess the ability to change in a certain wavelength range the topological charge of the incoming field by two units. In particular, we have also shown that such fibers can generate charge-2 optical vortices from the incoming Gaussian beams.

\section{References}

1. M. Vasnetsov and K. Staliunas, Optical Vortices, Vol. 228 of Horizons of World Physics (Nova Science, 1999).

2. M. S. Soskin and M. V. Vasnetsov, "Singular optics," Prog. Opt. 42, 219276 (2001).

3. M. W. Beijersbergen, L. Allen, H. E. L. O. van der Ween, and J. P. Woerdman, "Astigmatic laser mode converters and transfer of orbital angular momentum," Opt. Commun. 96, 123132 (1993).

4. M. W. Bejersbergen, R. P. C. Coerwinkel, M. Kristensen, and J. P. Woerdman, "Helical wavefront laser beams produced with a spiral phaseplate," Opt. Commun. 112, 321327 (1994).

5. V. Yu. Bazhenov, M. S. Soskin, and M. V. Vasnetsov, "Screw dis locations in light wavefronts," J. Mod. Opt. 39, 985990 (1992).

6. Ya. V. Izdebskaya, V. G. Shvedov, and A. V. Volyar, "Generation of higher order optical vortices by a dielectric wedge," Opt. Lett. 30, 24722474 (2005).

7. K. J. Webb and M. C. Yang, "Generation and control of optical vortices using left handed materials," Phys. Rev. E 74, 016601 (2006).

8. I. Skab, Y. Vasylkiv, B. Zapeka, V. Savaryn, and R. Vlokh, "Appearance of singularities of optical fields under torsion of crystals containing threefold symmetry axes," J. Opt. Soc. Am. A 28, 13311340 (2011).

9. L. Marrucci, C. Manzo, and D. Paparo, "Optical spin to orbital angular momentum conversion in inhomogeneous anisotropic media," Phys. Rev. Lett. 96, 163905 (2006).
10. L. Marrucci, E. Karimi, S. Slussarenko, B. Piccirillo, E. Santamato, E. Nagali, and F. Sciarrino, "Spin to orbital con version of the angular momentum of light and its classical and quantum applications," J. Opt. 13, 064001 (2011).

11. T. D. McGloin, N. B. Simpson, and M. J. Padgett, "Transfer of orbital angular momentum from a stressed fiber optic wave guide to a light beam," Appl. Opt. 37, 469472 (1998).

12. C. D. Poole, C. D. Townsend, and K. T. Nelson, "Helical grating two mode fiber spatial mode coupler," J. Lightwave Technol. 9, 598604 (1991).

13. K. S. Lee, "Coupling analysis of spiral fiber gratings," Opt. Commun. 198, 317324 (2001).

14. K. S. Lee and T. Erdogan, "Mode coupling in spiral fibre grat ings," Electron. Lett. 37, 156157 (2001).

15. K. S. Lee and T. Erdogan, "Fiber mode conversion with tilted gratings in an optical fiber," J. Opt. Soc. Am. A 18, 11761185 (2001).

16. C. N. Alexeyev, B. P. Lapin, and M. A. Yavorsky, "Helical core optical fibers maintaining propagation of a solitary optical vortex," Phys. Rev. A 78, 013813 (2008).

17. C. N. Alexeyev, B. P. Lapin, and M. A. Yavorsky, "The effect of spin orbit coupling on the structure of the stopband in heli cal core optical fibres," J. Opt. A: Pure Appl. Opt. 10, 085006 (2008).

18. C. N. Alexeyev, T. A. Fadeyeva, B. P. Lapin, and M. A. Yavorsky, "Generation of optical vortices in layered helical waveguides," Phys. Rev. A 83, 063820 (2011).

19. V. I. Kopp, V. M. Churikov, G. Zhang, J. Singer, C. W. Draper, N. Chao, D. Neugroschl, and A. Z. Genack, "Single and double helix chiral fiber sensors," J. Opt. Soc. Am. B 24, A48 A52 (2007).

20. S. Oh, K. R. Lee, U. C. Paek, and Y. Chung, "Fabrication of helical long period fiber gratings by use of a $\mathrm{CO}_{2}$ laser," Opt. Lett. 29, 14641466 (2004).

21. V. I. Kopp and A. Z. Genack, "Double helix chiral fibers," Opt. Lett. 28, 18761878 (2003).

22. C. N. Alexeyev and M. A. Yavorsky, "Optical vortices and the higher order modes of twisted strongly elliptical optical fi bres," J. Opt. A: Pure Appl. Opt. 6, 824832 (2004).

23. A. W. Snyder and J. D. Love, Optical Waveguide Theory (Chapman and Hall, 1985).

24. A. S. Davydov, Quantum Mechanics (Pergamon, 1976). 